\title{
Difficulty Weaning Off Cardiopulmonary Bypass: Role of Diabetes- Associated Cardiomyopathy
}

Thomas Nguyen (Resident) and Ahmed Zaky*

Department of Anesthesiology and Pain Medicine, University of Washington, USA

Keywords: Diabetes; Cardiomyopathy; Echocardiography; Cardiopulmonary bypass

\section{Introduction}

Diabetes mellitus is a progressive systemic disease that adversely affects cardiovascular function. Diabetes- associated cardiovascular changes are a major cause of morbidity and mortality worldwide. Clinical trials indicate that diabetes accelerates and worsens the prognosis of coronary atherosclerosis, is associated with a 3 -fold increase in the incidence of heart failure and that it is independently associated with worse heart failure prognosis [1]. The prognostic implications of thought of specific diabetes-induced structural, metabolic and functional cardiovascular changes triggered scientists to introduce the term (diabetic cardiomyopathy, DCM) as a separate clinical phenotype of myocardial dysfunction [2].

Coronary artery bypass grafting (CABG) remains the gold standard coronary revascularization procedure in patients with significant symptomatic obstructive coronary artery disease. Diabetics are thought to benefit from CABG given the multiplicity and extensiveness of their coronary atherosclerotic lesions [3]. On the other hand, diabetics are more prone to worse outcomes post cardiac surgery compared with others.

Despite of being a major risk factor of morbidity and mortality after cardiac surgery, a specific role of DCM has not been clearly elucidated.

This case reports a role that DCM might have played in adversely affecting a patient's course on CPB.

\section{Case Description}

A 51-year-old white male with an acute onset of chest discomfort and elevated troponins was taken to the operating room for a threevessel CABG. His past medical history was significant of type I diabetes on insulin (hemoglobin A-1C = $8 \mathrm{~g} / \mathrm{dl}$ ), hypothyroidism controlled by L-thyroxin, obesity (body mass index of 33), hypertension controlled by lisinopril and metoprolol, and smoking. Preoperative electrocardiogram (EKG) showed ST segments depressions $(>1 \mathrm{~mm})$ in the antero-spetal distribution. Preoperative transthoracic echocardiography (TTE) revealed normal left ventricular systolic function with an ejection fraction (EF) greater than $60 \%$, together with mild increase in left ventricular septalwall thickness $(1.3 \mathrm{~cm})$. Diastolic function was not reported on preoperative TTE. No other abnormalities were seen on TTE. Preoperative coronary angiography revealed significant triple vessel disease (left circumflex artery $=75 \%$, left anterior descending artery $=80 \%$, and posterior descending artery $=90 \%$ ). The patient was instructed to continue taking metoprolol and to abstain from taking lisinopril on the morning of the procedure.

Physical exam was significant of mild shortness of breath, tachycardia and mild sweating. Chest, airway and heart exam were unremarkable. Based on history and physical exam, the patient's calculated EUROSCORE II was 3.18.
In addition to standard ASA monitors, an arterial line catheter and a pulmonary artery catheter with continuous cardiac output monitoring capability were placed. General anesthesia was induced intravenously with ketamine/propofol (70 and $100 \mathrm{mg}$, respectively), fentanyl (250 $\mathrm{mcg}$ ), and succinylcholine (100 $\mathrm{mg})$. The trachea was intubated using a glidescope and a flexible fiberoptic scope due to an unanticipated difficulty in using direct laryngoscopy. Anesthesia was maintained with sevoflurane (1.5 MAC) in 50\% oxygen, and incremental doses of fentanyl and cisatracurium. Blood glucose levels were maintained in the range of $150-180 \mathrm{mg} / \mathrm{dl}$ by the use of insulin infusion. Despite a normal systolic function on preoperative TTE, intraoperative transesophageal echocardiography (TEE) (Siemens Acuson CV70) showed severely declining left ventricular global systolic function ( $\mathrm{EF}=35 \%$, using Simpson's method) and severe diastolic dysfunction (shown on TEE, Table 1). Due to severe systolic dysfunction, the patient was started on epinephrine infusion prior to initiation of $\mathrm{CPB}$ (Figure 1). Interestingly, despite normal biventricular end systolic and diastolic dimensions, small boluses of intravenous fluids ( $500 \mathrm{mls}$ ) were associated with noticeable increases in biventricular end diastolic areas and filling pressures (central venous and pulmonary end diastolic)on TEE and pulmonary artery catheter recordings, respectively.

A triple vessel bypass was performed successfully (left anterior descending artery to left internal mammary artery, saphenous venous graft to each of posterior descending artery and obtuse marginal arteries) was performed on CPB. Total aortic cross clamp and bypass times were 90 and 110 minutes, respectively.

Despite successful revascularization, the patient's biventricular cardiac systolic and diastolic functions continued to deteriorate (Figure 2). Inotropic agents (epinephrine and milrinone) were started at incremental doses (epinephrine up to $0.2 \mathrm{mcg} / \mathrm{kg} / \mathrm{min}$ and milrinone upto $0.375 \mathrm{mcg} / \mathrm{kg} / \mathrm{min}$ ) with no hemodynamic response. An intra-aortic balloon pump (maquet Datascope Corp CS100) was placed. Given the poor post-bypass ventricular function that was unresponsive to pharmacological inotropy and intra-aortic balloon counter-pulsation, a left ventricular assist device (LVAD) (HeartMate II, Thoratec Corp) was instituted. A postoperative coronary angiogram showed patent coronary grafts. Serum troponins remained elevated (three times normal values) in the postoperative period. The patient's

*Corresponding author: Ahmed Zaky, MD, MPH, Department of Anesthesiology and Pain Medicine, University of Washington, 1660 S Columbian way, S-112ANES, Seattle, WA 98108, USA, Tel: 206277 6723/352-217-6319; Fax: 206764 2914; E-mail: ahmed.zaky@va.gov

Received July 05, 2013; Accepted August 29, 2013; Published September 04 2013

Citation: Nguyen T, Zaky A (2013) Difficulty Weaning Off Cardiopulmonary Bypass: Role of Diabetes-Associated Cardiomyopathy. J Diabetes Metab 4: 287. doi:10.4172/2155-6156.1000287

Copyright: $\odot 2013$ Nguyen T, et al. This is an open-access article distributed under the terms of the Creative Commons Attribution License, which permits unrestricted use, distribution, and reproduction in any medium, provided the original author and source are credited. 


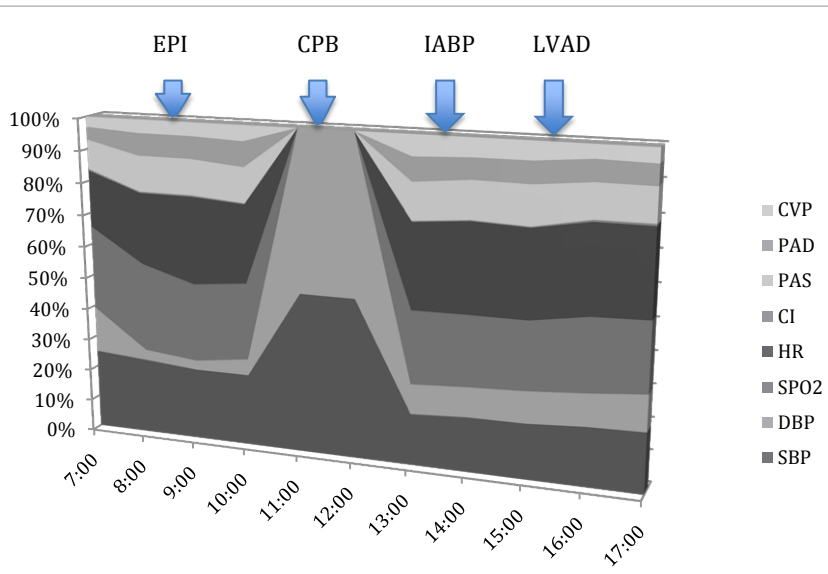

CPB: Cardiopulmonary Bypass; EPI: Epinephrine; IABP: Intra-Aortic Balloon Pump; LVAD: Left Ventricular Assist Device

Figure 1: A schematic diagram of hemodynamic data pre-, during, and post cardiopulmonary bypass. The $\mathrm{X}$-axis represents hourly time intervals. The $\mathrm{y}$-axis represents corresponding changes in hemodynamic data. Epinephrine was started to treat sever systolic dysfunction that stated after induction of anesthesia prior to CPB. An intra-aortic balloon pump was subsequently instituted. Left ventricular assist device was subsequently instituted due to a lack of pronounced hemodynamic response to pharmacologic and balloon pump support.

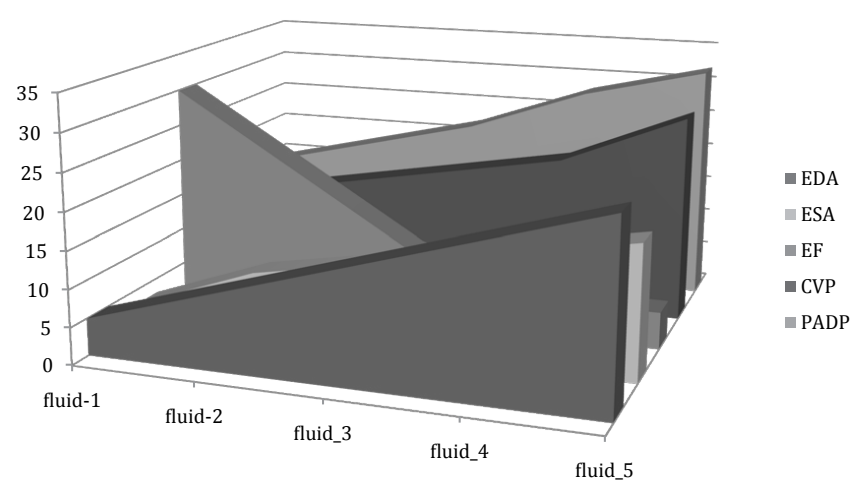

CVP: Central Venous Pressure; EDA: End Diastolic Area; ESA: End Systolic Area; EF: Ejection Fraction; PADP: Pulmonary Artery Diastolic Pressure

Figure 2: A schematic diagram showing the response of echocardiographic and hemodynamic indices to fluid bolus resuscitation, emphasizing the phenomenon of preload-intolerance. X-axis represents fluid bolus resuscitation. Y-axis represents response to fluid boluses. End diastolic, and end systolic areas, central venous and pulmonary artery diastolic pressures all increased, while ejection fraction decreased in response to fluid boluses.

myocardial function, however, continued to deteriorate and was not amenable to weaning from LVAD in the cardiac intensive care unit. Forty-eight hours later, the patient's care was deemed futile resulting in the family deciding to withdraw life support.

\section{Discussion}

This case report presents an atypical course pre-, during and post $\mathrm{CPB}$, in which a diabetic patient demonstrated a rapidly deteriorating and irreversible biventricular systolic and diastolic dysfunction despite successful revascularization. Furthermore, this case characteristically presents a phenomenon of 'preload intolerance' in which appropriate fluid boluses were associated with simultaneous biventricular dilatation and filling pressure elevations. A role of 'diabetes-associated cardiomyopathy' is strongly suggested.
Despite the coexistence of other comorbidities in our patient that could potentially explain the described course, a role for diabetes is strongly suggested. This is because as stated above, diabetes mutually hasten the acquisition and progression of heart failure, coronary artery disease, thyroid disease and hypertension; all being characterized with systolic as well as diastolic cardiac dysfunction. Moreover, despite the initial definition of 'diabetic cardiomyopathy' as a syndrome occurring exclusively in diabetics without coronary artery disease, there has never been a specific exclusive histologic (or clinical) diagnosis of DCM [4]. Moreover, many diabetics suffer from silent comorbidities that may not manifest clinically leading to a conception of an exclusive effect of diabetes on the heart in previously described reports of DCM. Besides, theoretically, adequate treatment of this patient's co-morbidities might have produced a controlled and less progressive state of secondary end organ damage. Taken together, given the fact that the effects of diabetes on the heart are neither mutually exclusive nor specific, we believe that a better term to describe those effects is 'diabetes-associated cardiomyopathy'.

Two-dimensional (2-D) and Doppler echocardiography was used in this case to show the combined systolic/diastolic dysfunction of diabetes associated cardiac dysfunction. In the presence of severe systolic dysfunction, trans-mitral flow pattern, as well as flow propagation velocity become more informative of impaired relaxation and elevated ventricular filling pressures compared with $\mathrm{E} / \mathrm{e}^{\prime}$. A restrictive diastolic pattern (Table 1) was shown on echocardiography denoting severe impairment of ventricular relaxation and reduction in compliance leading to an increase in atrial and filling pressures. Despite inherent technical limitations of 2-D echocardiographic modalities used in this case in terms of insonation angle-dependency, and the reliance of some indices (trans-mitral flow velocities, and pulmonary venous Doppler, PVD) on preload and heart rate [5], these indices have correlated well both diagnostically and prognostically with diastolic heart failure [6].

Diabetes affects the heart in multiple different ways; it may aggravate a pre-existing cardiac disease, it may induce a 'new' cardiac disease or it may potentiate the adverse cardiac effects of coexisting systemic diseases. In the presence of coexisting coronary artery disease, hypertension, obesity, hypothyroidism together with diabetes, our patient presents a combination of the three mechanisms.

Endothelial dysfunction is the hallmark of diabetic vasculopathy [7]. The elevated free fatty acids, insulin resistance, and increased production of advanced glycation end products (AGEs) seen with diabetes cause endothelial dysfunction. Endothelial dysfunction encompasses multiple abnormalities: altered vasomotor activity, vascular smooth cell dysfunction, overproduction of inflammatory cytokines and chemokines, impaired platelet function and abnormal coagulation. All of these abnormalities lead to increased vasoconstriction, inflammation and thrombosis [7].

Experimental evidence has shown that diabetes causes myocardial fibrosis cardiomyocyte hypertrophy, excitation-contraction decoupling, a shift in metabolic substrate from glucose to fatty acids, defects in intracellular calcium handling [8], and an increase in collagen formation and reactive oxygen species [9]. All these abnormalities result in functional and structural abnormalities noticeable clinically and by imaging techniques. Our patient suffered from obesity, hypothyroidism and dyslipidemia, all known to conspire with diabetes to worsen the metabolic substrate shift from glucose to free fatty leading to downstream impairment of intracellular calcium handling and 'lipid toxicity'[10]. 


\begin{tabular}{|c|c|c|c|c|}
\hline Parameter & TMDF & PVD & Color-M mode & TDI \\
\hline Witnessed value & $\begin{array}{l}-E / A_{R}>2 \\
-D T=125 \mathrm{msec} .\end{array}$ & $\begin{array}{l}-S / D<0.5 \\
-A_{r}=40 \mathrm{~cm} / \mathrm{s} \\
-A_{r}-A_{R}=20 \mathrm{msec}\end{array}$ & $\begin{array}{l}-V p=25 \mathrm{~cm} / \mathrm{s} \\
-E / V p=2.5\end{array}$ & $\begin{array}{l}\text { - Lateral e'= } 6 \mathrm{~cm} / \mathrm{s} \\
-\mathrm{E} / \mathrm{e}^{\prime}=14\end{array}$ \\
\hline Normal value (4) & $\begin{array}{l}-E / A=0.75-1.5 \\
-D T=140 \mathrm{msec}\end{array}$ & $\begin{array}{l}-S \geq D \\
-A_{r}<35 \mathrm{~cm} / \mathrm{s} \\
-A_{R} \text { dur }>A_{r}\end{array}$ & $\begin{array}{l}-\mathrm{Vp}=>55 \mathrm{~cm} / \mathrm{s} \\
-\mathrm{E} / \mathrm{Vp}=<1.5\end{array}$ & $\begin{array}{l}\text { - Lateral e'= } 8 \mathrm{~cm} / \mathrm{s} \\
-\mathrm{E} / \mathrm{e}^{\prime}=<10\end{array}$ \\
\hline
\end{tabular}

TMDF: Trans-Mitral Doppler Flow Velocity; PVD: Pulmonary Venous Doppler Flow Velocity; M-mode: Motion Mode; TDI: Tissue Doppler Imaging; E: Early Trans-Mitral Early Diastolic Rapid Ventricular Filling Velocity; DT: Deceleration Time from Peak to Baseline E Wave; A : Late Diastolic Atrial Reversal Contraction Velocity; S: Peak Systolic Pulmonary Venous Velocity; D: Early Pulmonary Venous Diastolic Velocity; A: Late Diastolic Atrial Reversal Pulmonary Venous Velocity; Vp= Intra-Ventricular Diastolic Flow Propagation Velocity; e': Lateral Mitral Annular Tissue Early Diastolic Velocity; E/e': Ratio of Trans-Mitral Early Diastolic Wave to Lateral Annular Mitral Tissue Early Diastolic Wave; dur: Duration

Table 1: Summary of echocardiographic findings witnessed in this case report.

Diastolic dysfunction in diabetes is thought to precede systolic dysfunction [11]. However, recent evidence suggests that certain sensitive indices of systolic myocardial deformation may be affected early as well [12]. The common denominator of both systolic and diastolic dysfunction is an earlier affection of the longitudinally arranged fibers that are more sensitive to ischemia and fibrosis than the circularly arranged fibers [13]. That is why tissue Doppler imaging of mitral annular motion may be affected earlier than EF. Systolic longitudinal strain measured by a novel echocardiographic technique (speckle tracking echocardiography) may detect earlier longitudinal axial dysfunction compared to tissue Doppler imaging given the insonation angle-independency of the former [12]. In our patient, reporting mitral annular tissue velocity, early trans-mitral flow or systolic longitudinal strain, might have unmasked an underlying myocardial dysfunction that was undetected by EF. The phenomenon of 'preload intolerance' noticed in our patient signifies a severe dysfunction in the longitudinal axial motion of both ventricles.

Diabetes is notorious of aggravating the severity and substantiating the risk of heart failure in patients with coronary artery disease [1]. Our patient had a history of long-standing diabetes, a factor that might have contributed to enlargement of the infarct size preoperatively, explaining the worsening of systolic function that was noticed prior to institution of CPB. Also, the resistance of systolic failure in the post bypass period to escalating doses of inotropes might be explained by impaired intracellular calcium handling in diabetes [14], the secondary messenger of intracellular inotropic action, together with impaired signaling of beta-adrenergic pathways [15]. A combination of epinephrine and milrinone was used to achieve inotropic synergism between both inotropes in addition to the lusitropic effects of milrinone given the degree of diastolic dysfunction witnessed in this case.

Diabetes worsens ischemia reperfusion injury and inhibits the protective effects of ischemia preconditioning [16]. The increase in reactive oxygen species from mitochondrial and extra-mitochondrial sources leads to direct damage to contractile proteins and potentiation of apoptosis [11]. This notion might explain why despite of successful revascularization and restoration of coronary blood flow, cardiac systolic and diastolic functions continued to deteriorate.

Generally speaking, our report goes in-line with other reports on the adverse effects of diabetes on outcomes post cardiac surgery such deep sternal wound infection [17], neurocognitive dysfunction [18] and kidney injury [19].

Novel imaging modalities have been described to diagnose subclinical cardiac dysfunction in patients with diabetes (with or without other co-morbidities), overcoming the conventionally used EF. Some of the limitations of EF include: load-dependency, lack of assessment of the function of the more vulnerable longitudinally arranged subendocardial fibers, and the inability to assess ventricular systolic dysfunction that is not associated with changes in ventricular cavity such as hypertrophic cardiomyopathy [20]. Assessment of cardiac mechanics along the longitudinal, radial and circumferential axes using speckle tracking echocardiography (STE) and cardiac magnetic resonance imaging (CMRI) has made it possible to overcome some of the limitations of conventionally used echocardiographic modalities used to diagnose systolic and diastolic cardiac dysfunction, such as load-, heart rate- and insonation angle-dependency. Strain and strain rate, torsion and synchrony are all examples of cardiac mechanics that can be sought using three dimensional speckle tracking (refer to use of ultrasonic markers that interact with ventricular tissue motion) and CMRI to detect regional and global systolic and diastolic cardiac dysfunction. The use of these modalities has shown superiority over conventional echocardiographic indices in multiple clinical trials [21-23].

Based on this case, it is appreciated that diabetes adversely affects cardiac function in cardiac surgical patients by myriad of mechanisms. As a result, diabetes should be more rigorously investigated in the preoperative evaluation of cardiac surgical patients. This entails the use of non-conventional imaging techniques (mentioned above), beyond what is conventionally performed for non-diabetic cardiac surgical patients. More powered studies are needed to investigate the prognostic implications of these measures, as well as the role of potential novel therapies in improving the outcome of diabetic cardiac surgical patients.

\section{References}

1. From AM, Leibson CL, Bursi F, Redfield MM, Weston SA, et al. (2006) Diabetes in heart failure: prevalence and impact on outcome in the population. Am J Med 119: $591-599$

2. Rubler S, Dlugash J, Yuceoglu YZ, Kumral T, Branwood AW, et al. (1972) New type of cardiomyopathy associated with diabetic glomerulosclerosis. Am J Cardiol 30: 595-602.

3. Javaid A, Steinberg DH, Buch AN, Corso PJ, Boyce SW, et al. (2007) Outcomes of coronary artery bypass grafting versus percutaneous coronary intervention with drug-eluting stents for patients with multivessel coronary artery disease. Circulation 116: I200-206.

4. Anderson KR, Sutton MG, Lie JT (1979) Histopathological types of cardiac fibrosis in myocardial disease. J Pathol 128: 79-85.

5. Daneshvar D, Wei J, Tolstrup K, Thomson LE, Shufelt C, et al. (2010) Diastolic dysfunction: improved understanding using emerging imaging techniques. Am Heart J 160: 394-404.

6. Rivas-Gotz C, Manolios M, Thohan V, Nagueh SF (2003) Impact of left ventricular ejection fraction on estimation of left ventricular filling pressures using tissue Doppler and flow propagation velocity. Am J Cardiol 91: 780-784.

7. Francesco Paneni, Joshua A. Beckman, Mark A. Creager, Francesco Cosentino (2013) Diabetes and vascular disease: pathophysiology, clinical consequences, and medical therapy: part I. Eur Heart J. 
8. Ganguly PK, Pierce GN, Dhalla KS, Dhalla NS (1983) Defective sarcoplasmic reticular calcium transport in diabetic cardiomyopathy. Am J Physiol 244: E528535.

9. Fischer VW, Barner HB, Larose LS (1984) Pathomorphologic aspects of muscular tissue in diabetes mellitus. Hum Pathol 15: 1127-1136.

10. An D, Rodrigues B (2006) Role of changes in cardiac metabolism in development of diabetic cardiomyopathy. Am J Physiol Heart Circ Physiol 291: H1489-1506.

11. Amour J, Kersten JR (2008) Diabetic cardiomyopathy and anesthesia: bench to bedside. Anesthesiology 108: 524-530.

12. Ernande L, Rietzschel ER, Bergerot C, De Buyzere ML, Schnell F, et al. (2010) Impaired myocardial radial function in asymptomatic patients with type 2 diabetes mellitus: a speckle-tracking imaging study. J Am Soc Echocardiogr 23: $1266-1272$

13. Miki T, Yuda S, Kouzu H, Miura T (2013) Diabetic cardiomyopathy: pathophysiology and clinical features. Heart Fail Rev 18: 149-166.

14. Ishikawa T, Kajiwara H, Kurihara S (1999) Alterations in contractile properties and $\mathrm{Ca} 2+$ handling in streptozotocin-induced diabetic rat myocardium. Am J Physiol 277: H2185-2194.

15. Amour J, Loyer X, Le Guen M, Mabrouk N, David JS, et al. (2007) Altered contractile response due to increased beta3-adrenoceptor stimulation in diabetic cardiomyopathy: the role of nitric oxide synthase 1-derived nitric oxide. Anesthesiology 107: 452-460.

16. Marso SP, Miller T, Rutherford BD, Gibbons RJ, Qureshi M, et al. (2007) Comparison of myocardial reperfusion in patients undergoing percutaneous coronary intervention in ST-segment elevation acute myocardial infarction with versus without diabetes mellitus (from the EMERALD Trial). Am J Cardiol 100: 206-210.

17. Kubota H, Miyata H, Motomura N, Ono M, Takamoto S, et al. (2013) Deep sternal wound infection after cardiac surgery. J Cardiothorac Surg 8: 132.

18. Toeg HD, Nathan H, Rubens F, Wozny D, Boodhwani M (2013) Clinical impact of neurocognitive deficits after cardiac surgery. J Thorac Cardiovasc Surg 145 : 1545-1549.

19. Vellinga S, Verbrugghe $W$, De Paep $R$, Verpooten GA, Janssen van Doorn $\mathrm{K}$ (2012) Identification of modifiable risk factors for acute kidney injury after cardiac surgery. Neth J Med 70: 450-454.

20. Kalogeropoulos AP, Georgiopoulou VV, Gheorghiade M, Butler J (2012) Echocardiographic evaluation of left ventricular structure and function: new modalities and potential applications in clinical trials. J Card Fail 18: 159-172.

21. Park SJ, Miyazaki C, Bruce CJ, Ommen S, Miller FA, et al. (2008) Left ventricular torsion by two-dimensional speckle tracking echocardiography in patients with diastolic dysfunction and normal ejection fraction. J Am Soc Echocardiogr 21: 1129-1137.

22. Nelson MD, Haykowsky MJ, Petersen SR, DeLorey DS, Cheng-Baron J, et al (2010) Increased left ventricular twist, untwisting rates, and suction maintain global diastolic function during passive heat stress in humans. Am J Physio Heart Circ Physiol 298: H930-937.

23. Tan YT, Wenzelburger F, Lee E, Heatlie G, Leyva F, et al. (2009) The pathophysiology of heart failure with normal ejection fraction: exercise echocardiography reveals complex abnormalities of both systolic and diastolic ventricular function involving torsion, untwist, and longitudinal motion. J Am Coll Cardiol 54: 36-46. 